\title{
Biomedical image retrieval using microscopic configuration with local structural information
}

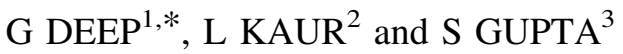 \\ ${ }^{1}$ Department of Computer Science and Engineering, Chandigarh Engineering College (CEC), Landran, Punjab \\ Technical University, Mohali 140307, India \\ ${ }^{2}$ Department of Computer Engineering, Punjabi University, Patiala 147002, India \\ ${ }^{3}$ Department of Computer Science and Engineering, University Institute of Engineering and Technology, Panjab \\ University, Chandigarh 160014, India \\ e-mail: gaganpec@yahoo.com; mahal2k8@gmail.com; savita2k8@yahoo.com
}

MS received 16 April 2015; revised 15 June 2016; accepted 4 August 2017; published online 10 March 2018

\begin{abstract}
This paper focusses on the use of microscopic configuration (MiC) for discriminative information to retrieve lung cancer images. Existing local binary pattern $(L B P)$ detects the local structures, such as lighting spots and edges in images, whereas the local configuration pattern $(L C P)$ explores multi-channel discriminative information of both the $\mathrm{MiC}$ and local structures of images. Both methods are used to extract the rotation- and scale-invariant features from all lung cancer images. The performance of these methods is tested by conducting experiments on benchmark biomedical database of Lung Image Database Consortium and Image Database Resource Initiative-computer tomography. The database includes CT images with region of interest. The results show that $L C P$ yields significant improvement in terms of average retrieval precision and average retrieval rate as compared with $L B P$ and other state-of-the-art texture descriptors.
\end{abstract}

Keywords. Image retrieval; local binary pattern; local configuration pattern; medical imaging; texture.

\section{Introduction}

Day by day, the importance of medical images is increasing in medical hospitals for patient diagnosis. The medical image data exist in different formats such as computer tomography (CT), magnetic resonance imaging (MRI), ultrasound (US) and X-ray. As we know that medical imaging is made up of dissimilar minor structures, there has been much interest of researchers in the development of well-structured techniques to work on huge image databases of biomedical images for efficient access, search and retrieval. To address this problem, content-based biomedical image retrieval came into existence. The content-based image retrieval (CBIR) uses the visual content features such as colour, texture, shape, spatial layout, etc. of regions or objects to represent and index the biomedical image database for efficient retrieval. The previously available image retrieval systems are presented by Giuliano et al [1], Michalis et al [2], An et al [3], Gianluca et al [4], Sihyoung et al [5] and Yue et al [6].

The main step of the CBIR is feature extraction, the effectiveness of which rests upon the method derived for extracting features from given images. The visual content descriptors are either global or local. A global descriptor

*For correspondence represents the visual features of the whole image, whereas a local descriptor represents the visual features of regions or objects to describe the image. These features are arranged as multi-dimensional feature vectors and stored in the feature database. The selection of feature descriptors affects the image retrieval performance.

In early years, Manjunath et al [7] used features based on intensity histogram for biomedical image retrieval. However, their retrieval performance is usually limited especially on large databases due to lack of discrimination power of such descriptors. To address this problem, texture-based features have been proposed for biomedical image retrieval. They are extensively used in the image processing applications to identify the visual patterns. Most of the biomedical images represented in greyscale are extensively textured in common. Hence, in clinical exams, the appearance of organ/tissue/lesion in the images is due to intensity variations laid on various texture characteristics. Thereafter, texture became very popular in biomedical image retrieval because of eminent significance of acquired texture. For the texture analysis, the local binary pattern $(L B P)$ by Ojala et al $[8]$ is proposed. $L B P$ has low computational complexity and capacity of coding minute specifications. Ojala et al [9] advocated some more moderations in $\angle B P$ for texture classifications. In the field of medicine, Oliver et al [10] used $L B P$ descriptor for mammogram images and Unay and Ekin [11] introduced a 


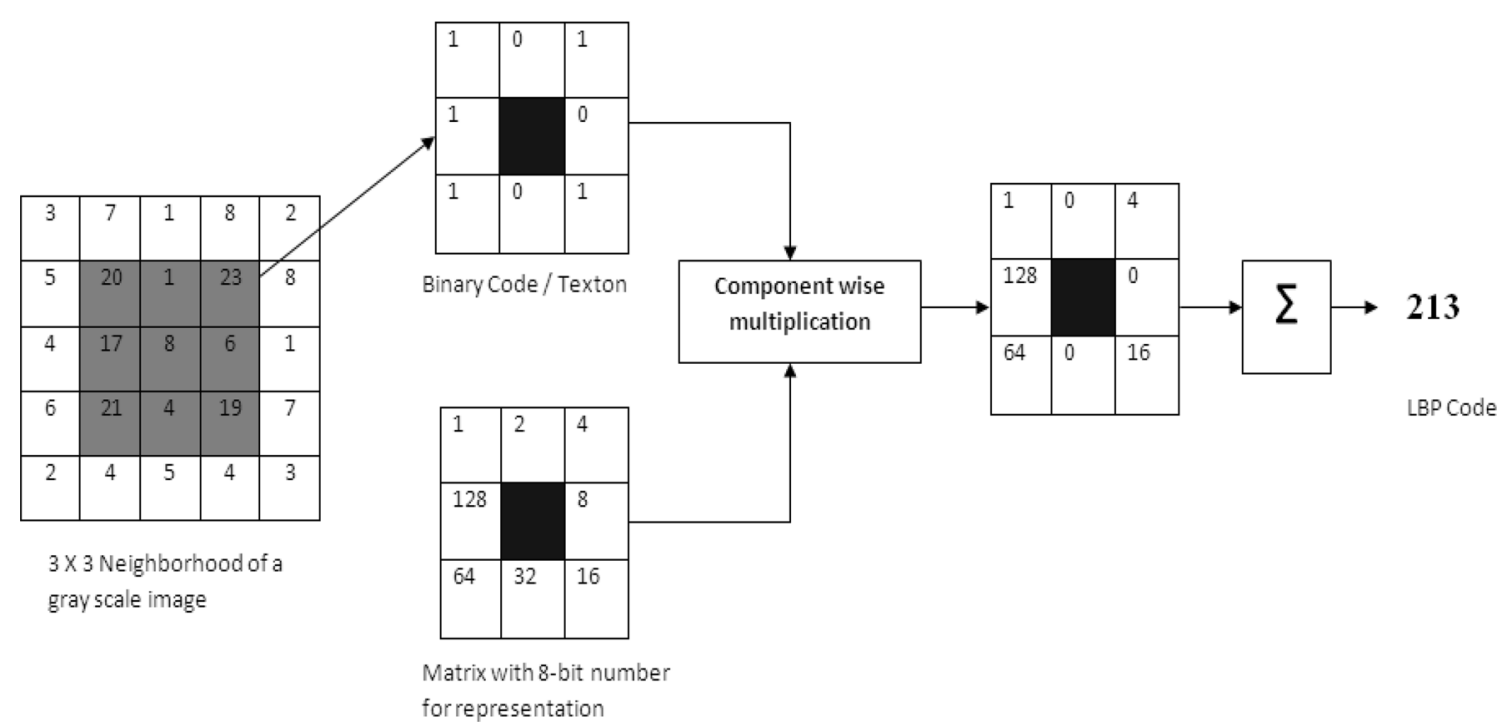

Figure 1. Example of obtaining LBP for $3 \times 3$ neighbourhoods (adopted from Ojala et al [9]).

powerful search and retrieval method that used $L B P$ with Support Vector Machines (SVMs) to find relevant slices in brain magnetic resonance volumes. Keramidas et al [12] have given texture representation on thyroid US images. Nanni and Lumini $[13,14]$ used $L B P$ to automate the cell phenotype image classification. In the area of face classification, Nanni and Lumini [15] and Ahonen et al [16] have extensively worked on $L B P$. Guo et al [17] proposed the rotation-invariant $L B P$ variance operator for texture classification. In some important works, Nanni and Lumini [18] and Shang and Veldhuis [19] have also successfully experimented $L B P$ in other useful applications. More on $L B P$ can be explored at http://www.ee.oulu.fi/mvg/page/lbp_bibliography \#biomedical.

For richer and more detailed information from normal texture databases, Guo et al [20] proposed local configuration pattern $(L C P)$ by integrating the microscopic configuration (MiC) information with local structural information of $L B P$ descriptor. Image $\mathrm{MiC}$ contains robust and reliable discriminative information, which has improved the classification performance. The robustness of $L C P$ s against illumination variations and image rotation is strongly implied. In literature, Murala et al [21-24] and Murala and Jonathan $[25,26]$ had proposed many extended versions of $L B P$ to derive new image features for biomedical image indexing and retrieval. In this paper, discrimination power of $\angle B P$ and $L C P$ has motivated us to use the biomedical images for image retrieval.

The organization of the paper is as follows. In section 1, a brief review of medical image retrieval and related work is given. A concise review of local patterns can be further visualized in section 2. The retrieval system analysis and framework of the existing descriptors are presented in section 3. Experimental results and discussion are presented in section 4 . Finally, the concluding remarks are given in section 5.

\section{Local patterns}

\subsection{LBP formulation}

The $L B P$ was first introduced by Ojala et al [9] for rotationinvariant texture classification. The $L B P$ is a very efficient texture descriptor due to its discriminative power and computational simplicity. $I$ is a greyscale image of size $n \times m$ pixels and $I(g)$ denotes the grey level of the $g$ th pixel in the image $I$. The $L B P$ operator is calculated at each pixel by evaluating the binary differences of the values in a small circular neighbourhood (with radius $R$ ) around the value of a central pixel, $g_{c}$. Mathematically, the $L B P$ value of current pixel is

$$
L B P_{P, R}=\sum_{p=0}^{P-1} f_{1}\left(g_{p}-g_{c}\right) 2^{p}, f_{1}(x)= \begin{cases}1 & \text { if } x \geq 0 \\ 0 & \text { otherwise }\end{cases}
$$

where $g_{c}$ is the grey value of the central pixel, $g_{p}$ are the grey values of the circularly symmetric neighbourhood $g_{p}$ $(p=0, \ldots, P-1), P$ is the image pixels in the circle of radius $R(R>0)$ and $2^{p}$ is the binomial factor for each sign $f_{1}\left(g_{p-} g_{c}\right)$.

A histogram is generated to represent the texture image after finding the $L B P$ code of each pixel in the image. Figure 1 shows how a texton and $L B P$ code are derived; the $L B P$ takes the $3 \times 3$ neighbourhood of a central pixel and generates a binary 1 if the neighbour of that pixel has a larger value; otherwise, it produces a binary 0 . An $L B P$ code for a neighbourhood is produced by multiplying the threshold values with weights given to the corresponding pixels, and summing up the result. Thus, each $L B P$ is regarded as a micro-texton in Ojala et al [9].

Local textons include spots, flat areas, edges, line ends and corners. Figure 2 shows the different texture primitives 

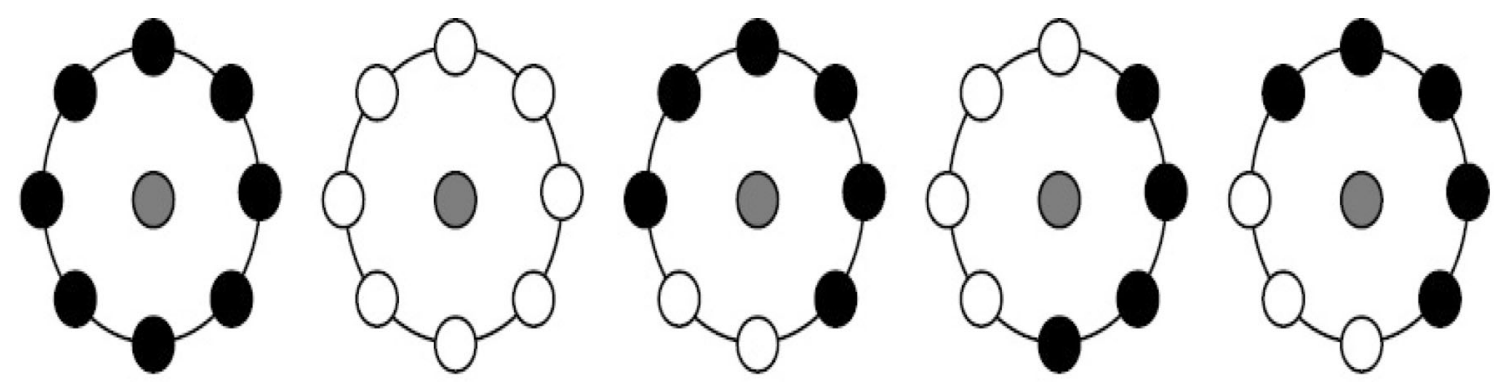

Figure 2. Different texture primitives (spot, spot/flat, line end, edge and corner) detected by the uniform patterns of $L B P(\mathrm{Ojala}$ et al [9]).

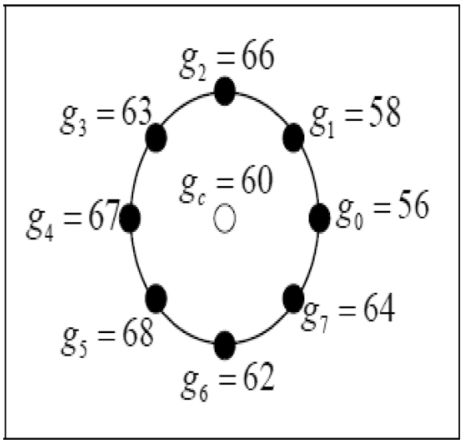

(a)

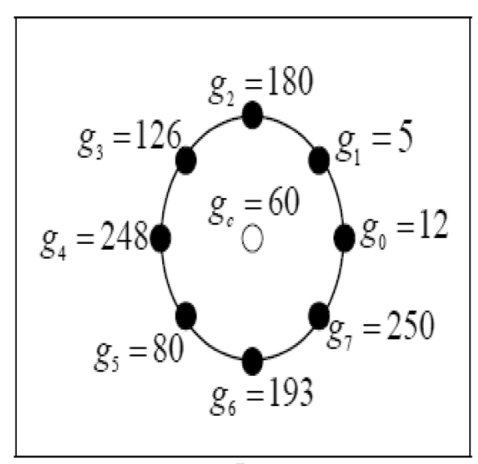

(b)

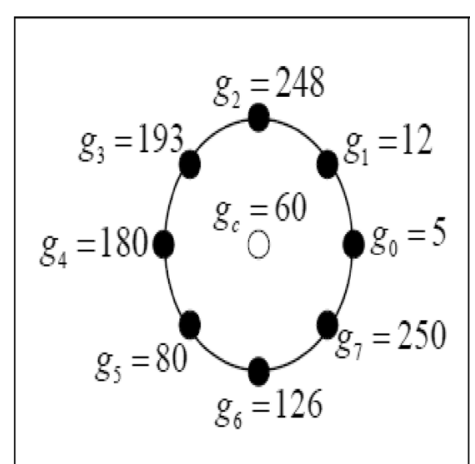

(c)

Figure 3. Sample patterns to analyse the performance: intensity variations $\left(g_{c}-g_{i}\right)$ between the central pixel and neighbourhood. Here, $g_{c}$ denotes the intensity of the central pixel and $g_{i}(i=0, \ldots, 7)$ denotes the intensities of neighbourhood pixels.

detected by the uniform patterns of $L B P$. In the figure, grey circle indicates the central pixel, black and white circles correspond to bit values of 0 and 1 in the different patterns of the operator.

2.1a Rotation invariance [9]: To remove the effect of rotation, each $L B P$ code must be rotated back to a reference position, effectively making all rotated versions of a binary code the same. This transformation is defined as follows:

$$
L B P_{P, R}^{r i}=\min \left\{R O R\left(L B P_{P, R}, i\right) \mid i=0, \ldots, P-1\right\}
$$

where the superscript ' $r i$ ' stands for 'rotation invariant'; the function $\operatorname{ROR}(x, i)$ performs ' $i$ ' times bit-wise circular right shift on the $P$-bit binary number, $x$. In terms of image pixels, this simply corresponds to rotating the neighbour set clockwise so as to get maximum number of zeros in the beginning of the binary string; 'min' operator will just take the minimum decimal values from different patterns.

2.1b Uniform pattern [9]: Sometimes, rotation-invariant patterns cannot give good discrimination. Some patterns among rotation-invariant patterns are dominant, and are fundamental properties of texture. The patterns are considered uniform if the number of transitions between 0 and 1 is less than or equal to two in the circular binary code. This operator is called $L B P_{P, R}^{r i u 2}$. Patterns are considered non- uniform if they contain the main part of the noise of the images. A region with no transitions is considered as a background or a flat region of the image. The $L B P$ feature vector is extracted from each cell and is the histogram of dimension $P+2$ (a single bin for non-uniform patterns). $L B P_{P, R}^{r i u 2}$ operator is formally defined as follows:

$$
L B P_{P, R}^{r i u 2}= \begin{cases}\sum_{p=0}^{P-1} s\left(g_{p}-g_{c}\right) & \text { if } U\left(L B P_{P, R}\right) \leq 2 \\ P+1 & \text { otherwise }\end{cases}
$$

where

$$
\begin{aligned}
U\left(L B P_{P, R}\right)= & \left|s\left(g_{P-1}-g_{c}\right)-s\left(g_{0}-g_{c}\right)\right| \\
& +\sum_{p=0}^{P-1}\left|s\left(g_{p}-g_{c}\right)-s\left(g_{p-1}-g_{c}\right)\right|
\end{aligned}
$$

where superscript 'riu2' denotes the rotation-invariant 'uniform' patterns that have $U$ values that are at most 2 . Therefore, mapping from $L B P_{P, R}$ to $L B P_{P, R}^{r i u 2}$ results in only $p+1$ distinct groups of patterns, leading to a much shorter histogram representation for the whole image. To achieve rotation invariance, a Look-Up Table (LUT) is used. The LUT stores all the possible uniform patterns along with their unique $L B P_{8, R}^{r i u 2}$ code. 
In conventional $L B P$ the central pixel is discarded (despite the implicit use of the intensity of the central pixel as the threshold to achieve local grey-scale invariance), and only the joint distribution of the neighbourhood around each pixel is considered. The $L B P$ oversimplifies local structure and loses textural information. Therefore, Ojala et al [9] made a correction by including the local contrast of each pattern and proposing a complementary local descriptor called $V A R_{P, R}$. Using the joint histogram of $L B P_{P, R}^{r i u 2}$ and $V A R_{P, R}$, denoted as $L B P_{P, R}^{r i u 2} / V A R_{P, R}$ (aka $L B P V$ ), a powerful tool for rotation-invariant texture classification is

$$
V A R_{P, R}=\frac{1}{P} \sum_{p=0}^{P-1}\left(g_{p}-\mu\right)^{2}, \text { where } \mu=\frac{1}{P} \sum_{p=0}^{P-1} g_{p}
$$

\section{$2.2 \operatorname{LCPs}[20]$}

Guo et al [20] proposed an $L C P$ descriptor for texture image description by combining information with $L B P$. The descriptor provides the information of images in two parts: (1) local structural information and (2) MiC information that involves image configuration and pixel-wise interaction relationships. $L B P$ is used to extract the local structural information and construct pattern occurrence histograms of images, whereas $\mathrm{MiC}$ information is explored by encoding linear relationships among neighbouring pixels. However, $L B P$-based local features show the same pattern type of figure $3 \mathrm{a}$ and $\mathrm{b}$ while their textural surfaces are totally different from each other.

By incorporating $L B P$ with local variance information [9], patterns could be distinguished, while patterns in figure $3 \mathrm{~b}$ and $\mathrm{c}$ would still be considered as the same pattern type because of the same variance. However, they are actually two patterns with different textural properties. Therefore, higher discriminator power than local variance is included by encoding both the magnitude information and pixel-wise interaction relationships for effective image representation in the retrieval framework.

2.2a Discriminative representation by microscopic information (adopted from Guo et al [20]): We aim to model pixel-wise interaction relationships with respect to each rotation-invariant uniform pattern. To be specific, we estimate optimal weights associated with intensities of neighbouring pixels to linearly reconstruct the central pixel intensity for each pattern type. This is expressed by the relation

$$
E\left(a_{0, \ldots,}, a_{P-1}\right)=\left|g_{c}-\sum_{i=0}^{P-1} a_{i} g_{i}\right|
$$

In this formula, $a_{i}(i=0, \ldots, P-1)$ are weighting parameters associated with $g_{i}$, and $E\left(a_{0}, \ldots, a_{P-1}\right)$ is the reconstruction error regarding model parameters $a_{i}(i=0$, $\ldots, P-1)$. To minimize the reconstruction error for each pattern, optimal parameters are determined by the least squares estimation as presented by Roweis and Saul [27].

Least squares estimation: For a given image $I$, suppose the occurrence of a particular pattern type $L$ is $N_{L}$, which means there are $N_{L}$ pixels in $I$ with the pattern type $L$. The label code is calculated by Eq. (1) or (3), depending on what kind of patterns is of interest. We denote the intensities of these $N_{L}$ pixels as $c_{L, i}\left(i=0, \ldots, N_{L}-1\right)$, and organize them into a vector:

$$
C_{L}=\left(\begin{array}{c}
C_{L, 0} \\
C_{L, 1} \\
\vdots \\
\cdot \\
C_{L, N_{L}-1}
\end{array}\right)
$$

The intensities of their neighbouring pixels $v_{i} ; 0, \ldots$, $v_{i} ; \mathrm{P}-1\left(i=0, \ldots, N_{L}-1\right)$ can thus be organized as follows:

$$
V_{L}=\left(\begin{array}{c}
v_{0 ; 0} v_{0 ; 1} \ldots v_{0 ; P-1} \\
v_{1 ; 0} v_{1 ; 1} \ldots v_{1 ; P-1} \\
: \\
\cdot \\
v_{N_{L}-1 ; 0} v_{N_{L}-1 ; 1} \ldots v_{N_{L}-1 ; P-1}
\end{array}\right)
$$

In order to minimize the reconstruction error in Eq. (6), the unknown parameters $a_{i}(i=0, \ldots, P-1)$ are constructed as a column vector:

$$
A_{L}=\left(\begin{array}{c}
a_{0} \\
a_{1} \\
\vdots \\
\cdot \\
a_{P-1}
\end{array}\right)
$$

In this way, the problem becomes a least-square problem $C_{L}=V_{L} A_{L}$. When the system is over-determined, optimal parameter vector $A_{L}$ is determined by the relation

$$
A_{L}=\left(V_{L}^{T} V_{L}\right)^{-1} V_{L}^{T} C_{L}
$$

Otherwise, when $N_{L} \leq P$, the pattern $L$ rarely occurs; hence, it would be considered as a non-reliable pattern to serve as a feature. In this case, each entry of the parameter vector will be set to zero. By considering the pixel-wise interaction, the parameter vectors of different patterns shown in figure $3 \mathrm{~b}$ and $\mathrm{c}$ would not be the same since each entry of $A_{L}$ changes according to the relative neighbourhood positions. Therefore, the obtained feature is capable of capturing both the information of magnitude and pixel-wise interactions, which makes it more discriminative than the previous representation.

Rotation-invariant analysis: In the area of texture analysis, rotation-invariant analysis is a widely studied problem, which aims at providing texture features that are invariant to rotation angle of the input image. To produce 


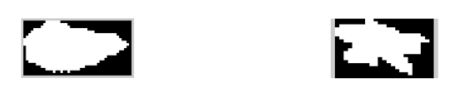

Sample Image $1 \quad$ Sample Image 2

(a)

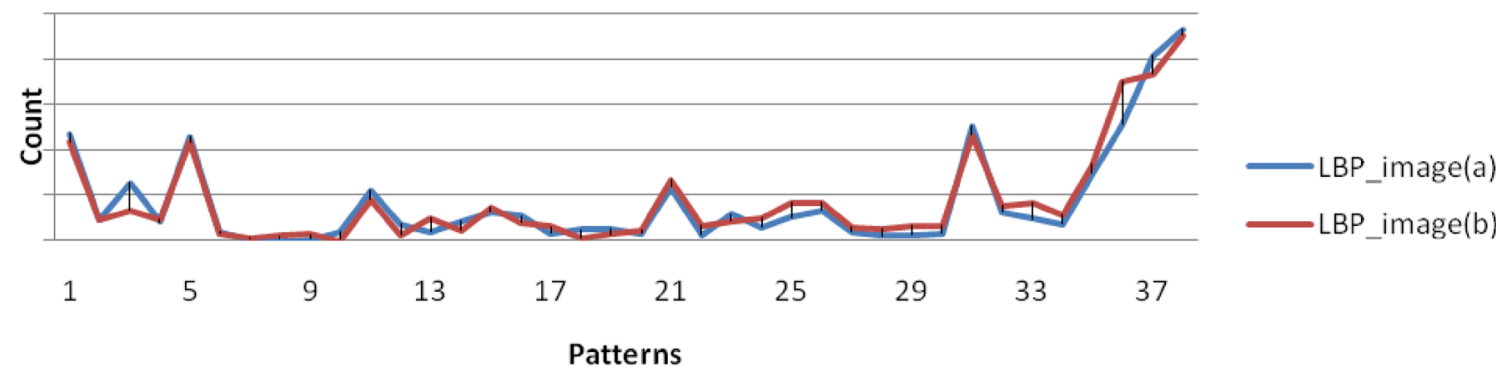

(b)

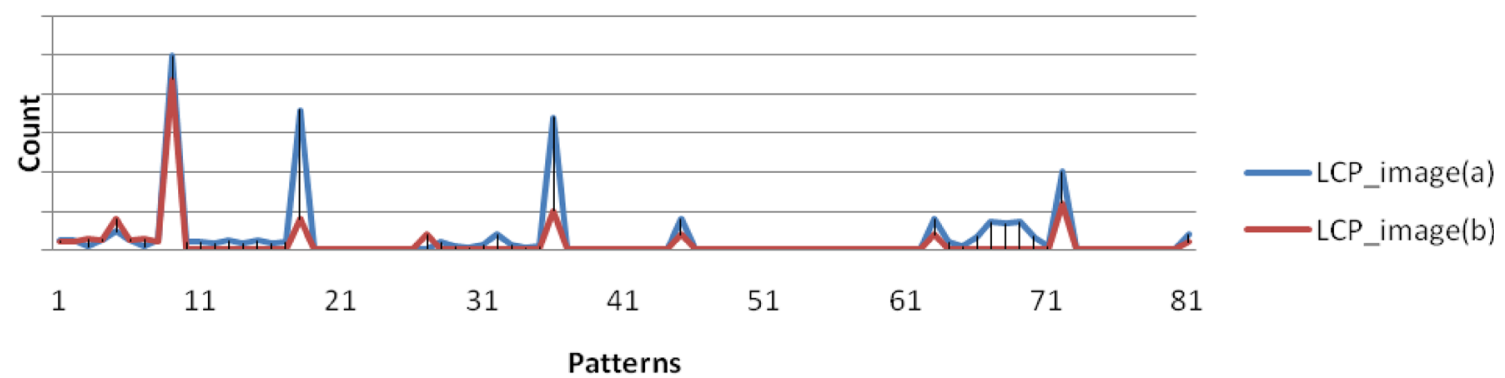

(c)

Figure 4. (a) Two sample images. (b, c) Comparison of various features on two sample images from LIDC-IDRI-CT database.

rotation-invariant features, we apply 1D Fourier transform to the estimated parameter vector $A_{L}$. The transformed vector is expressed by the relation

$$
H_{L}(k)=\sum_{i=0}^{P-1} A_{L}(i) e^{-\frac{j 2 \pi k i}{P}}
$$

where $H_{L}(k)$ is the $k^{\text {th }}$ element of $H_{L}$ and $A_{L}(i)$ is the $i$ th element of $A_{L}$. Although image rotation would lead to cyclic translations of $A_{L}$, Fourier transform is invariant to this kind of translations so that $H_{L}$ can achieve rotationinvariant property. The magnitude part of vector $H_{L}$ is taken as the MiC feature, which is defined by the relation

$$
\left|H_{L}\right|=\left[\left|H_{L}(0)\right| ;\left|H_{L}(1)\right| ; \ldots ;\left|H_{L}(P-1)\right|\right] .
$$

Considering that $\left|H_{L}\right|$ encodes the image configuration and pixel-wise interaction relationship of each specific pattern, it together with pattern occurrences of $L B P$ s would construct a complementary feature for both the discrimination of $\mathrm{MiC}$ and local structures. In this way, the final feature is

$$
L C P=\left[\left[\left|H_{0}\right| ; O_{0}\right] ;\left[\left|H_{1}\right| ; O_{1}\right] ; \ldots ;\left[\left|H_{q-1}\right| ; O_{q-1}\right]\right]
$$

where $\left|H_{i}\right|$ is calculated by Eq. (12) with respect to the $i$ th pattern of interest, $\left|O_{i}\right|$ is the occurrence of the $i$ th local pattern of interest (i.e., the $L B P$ ) and $q$ is the total number of patterns of interest. Moreover, multi-scale analysis is achieved by combining $L C P$ s with different radii and neighbouring samples.

\section{Retrieval analysis and framework}

In the literature, it is observed that the pattern-based features are analysed on normal texture databases. Hence, in this paper, the effectiveness of $L C P$ over $L B P$ and other existing texture patterns is analysed by conducting experiments on biomedical databases.

\subsection{Analysis}

Figure 4 illustrates the comparison between the various features on two sample images as shown in figure $4 \mathrm{a}$, which are selected from the different categories of Lung Image Database Consortium and Image Database Resource Initiative (LIDC-IDRI)-CT database. By visualization we can identify that the sample images are not similar. The histograms of both images (two sample images) using $L B P$ methods as in figure $4 \mathrm{~b}$ are almost similar. However, the histograms of both images using $L C P$ are not similar, which 


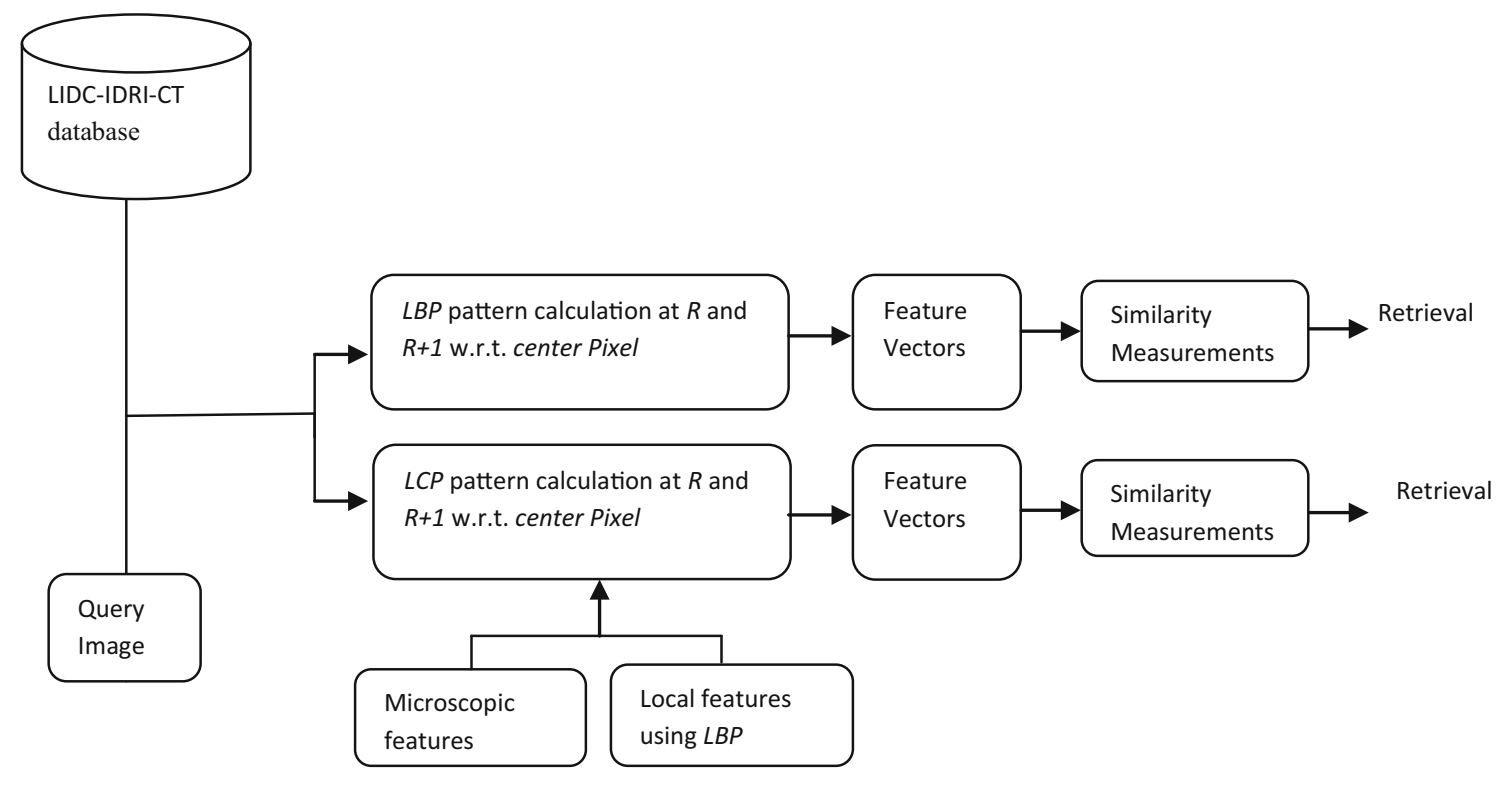

Figure 5. Retrieval framework.

can be seen in figure 4c. From figure 4, it is clear that the $L C P$ method is able to differentiate the two sample images as compared with $L B P$.

\subsection{System framework}

Figure 5 illustrates the framework of the proposed analysis system and algorithm for the same is given as follows.

\section{Algorithm.}

Input: Query image;

Output: Retrieval results

1. Load the query image.

2. Calculate the $L B P$ features at $R$ and $R+1$ with respect to the central pixel.

3. Also calculate the $L C P$ features at $R$ and $R+1$ with respect to the central pixel.

4. Form feature vectors of calculated values from $L B P$ and $L C P$.

5. First, using $L B P$ feature vectors of images, compare the query image with the images in the database using similarity measure.

6. Second, using $L C P$ feature vectors, compare the query image with the images in the database using similarity measure.

7. Retrieve the images based on the best matches.

This algorithm is applied on the database LIDC-IDRI-CT for best matches. Finally, results from these two methods are compared.

\subsection{Similarity measure}

The representation of feature vector of query image $Q$ is $f_{Q}=\left(f_{Q_{1}}, f_{Q_{2}}, \ldots, f_{Q_{L_{B}}}\right)$ acquired after the feature extraction. Likewise, dataset $|D B|$ of all medical images is represented with feature vectors $f_{D B_{j}}=\left(f_{D B_{j 1}}, f_{D B_{j 2}}, \ldots, f_{D B_{j L_{g}}}\right) ; j=1,2, \ldots,|D B|$. However, the only purpose here is to obtain $n$ top match images by measuring the distance between query image and image in the dataset $|D B|$. Finally, $n$ best images are displayed that are similar to the query image.

In this paper, Euclidean distance measure is used as similarity distance metrics and this is shown as follows:

$$
D(Q, D B)=\left(\sum_{i=1}^{L_{g}}\left(f_{D B_{j i}}-f_{Q, i}\right)^{2}\right)^{\frac{1}{2}}
$$

where $f_{D B_{j i}}$ is the $i$ th feature of the $j$ th image in the database $|D B|$.

\subsection{Evaluation measures (adopted from Murala and Jonathan [25])}

For performance evaluation, the average retrieval precision $(A R P)$ and average retrieval rate $(A R R)$ are used. Murala and Jonathan [25] defined the precision $(P)$ and recall $(R)$ for the query image $I_{q}$, as follows:

$$
\text { precision: } P\left(I_{q}\right)=\frac{\text { number of relevant images retrieved }}{\text { total number of images retrieved }}
$$




$$
A R P=\left.\frac{1}{|D B|} \sum_{i=1}^{|D B|} P\left(I_{i}\right)\right|_{n \leq 10}
$$

$$
\begin{aligned}
\text { recall }: & R\left(I_{q}\right) \\
& =\frac{\text { number of relevant images retrieved }}{\text { total number of relevant images in the database }}
\end{aligned}
$$

$$
A R R=\left.\frac{1}{|D B|} \sum_{i=1}^{|D B|} R\left(I_{i}\right)\right|_{n \geq 10}
$$

\subsection{Abbreviations}

The abbreviations used in the analysis are the following:

\section{$L B P$}

$L B P_{1,8}^{u 2}$

$L B P_{1,8}^{u 2} / V A R_{1,8}$

$\operatorname{LBP} P_{1,8}^{\text {riu } 2}$ local binary patterns as defined in Ojala et al [8,9]

$L B P$ with uniform patters at $(1,8)$ neighbourhood size

$L B P$ with uniform patters at $(1,8)$ neighbourhood size with variance information

$L B P$ with rotation-invariant uniform patters at $(1,8)$ neighbourhood size
$\operatorname{LBP}_{1,8}^{\text {riu } 2} / V A R_{1,8}$

$L P Q$

$L Q P$

$B G P$

$B S I F$

$W L D$

SIFT

GLCM

$L C P$

$\operatorname{LCP}_{1.8}^{\text {riu } 2}$

$\operatorname{LCP}_{2,8}^{\text {riu } 2}$

$L C P_{2,16}^{\text {riu2 }}$
$L B P$ with rotation-invariant uniform patters at $(1,8)$ neighbourhood size with variance information

local phase quantization as defined in Ojansivu and Heikkila [28]

local quinary patterns as defined in Nanni et al [29]

binary Gabor patterns as defined in Zhang et al [30]

binarized statistical image features as defined in [31]

Weber law descriptor as defined in Chen et al [32]

scale-invariant feature transform as defined in Lowe [33]

Grey level co-occurrence matrix as defined in Haralick et al [34]

Local configuration patters as defined in Guo et al [20]

$L C P$ with rotation-invariant uniform patters at $(1,8)$ neighbourhood size $L C P$ with rotation-invariant uniform patters at $(2,8)$ neighbourhood size

\begin{tabular}{|c|c|c|c|c|c|c|c|}
\hline $\begin{array}{l}\text { Case } \\
\text { no. }\end{array}$ & Data & No. of slices & $\begin{array}{l}\text { No. of } \\
\text { images }\end{array}$ & Resolution & $\begin{array}{l}\text { Slice thickness } \\
(\mathrm{mm})\end{array}$ & $\begin{array}{c}\text { Tube voltage } \\
(\mathrm{kV})\end{array}$ & $\begin{array}{c}\text { Tube current } \\
(\mathrm{mA})\end{array}$ \\
\hline 1 & $\begin{array}{c}\text { LIDC-IDRI- } \\
0002\end{array}$ & 20 & 40 & $512 \times 512$ & 1.3 & 120 & 440 \\
\hline 2 & $\begin{array}{l}\text { LIDC-IDRI- } \\
0003\end{array}$ & 10 & 40 & $512 \times 512$ & 2.5 & 120 & 300 \\
\hline 3 & $\begin{array}{c}\text { LIDC-IDRI- } \\
0006\end{array}$ & 20 & 80 & $512 \times 512$ & 1.3 & 120 & 440 \\
\hline 4 & $\begin{array}{c}\text { LIDC-IDRI- } \\
0007\end{array}$ & 21 & 84 & $512 \times 512$ & 1.3 & 120 & 440 \\
\hline 5 & $\begin{array}{c}\text { LIDC-IDRI- } \\
0010\end{array}$ & 15 & 60 & $512 \times 512$ & 1.3 & 120 & 401 \\
\hline 6 & $\begin{array}{c}\text { LIDC-IDRI- } \\
0011\end{array}$ & 27 & 108 & $512 \times 512$ & 2.5 & 120 & 265 \\
\hline 7 & $\begin{array}{c}\text { LIDC-IDRI- } \\
0012\end{array}$ & 20 & 80 & $512 \times 512$ & 2.5 & 120 & 300 \\
\hline 8 & $\begin{array}{c}\text { LIDC-IDRI- } \\
0013\end{array}$ & 18 & 72 & $512 \times 512$ & 2.5 & 120 & 320 \\
\hline 9 & $\begin{array}{c}\text { LIDC-IDRI- } \\
0014\end{array}$ & 07 & 28 & $512 \times 512$ & 2.5 & 120 & 300 \\
\hline 10 & $\begin{array}{c}\text { LIDC-IDRI- } \\
0015\end{array}$ & 19 & 76 & $512 \times 512$ & 1.3 & 120 & 361 \\
\hline 11 & $\begin{array}{c}\text { LIDC-IDRI- } \\
0016\end{array}$ & 26 & 104 & $512 \times 512$ & 2.5 & 120 & 265 \\
\hline 12 & $\begin{array}{c}\text { LIDC-IDRI- } \\
0017\end{array}$ & 26 & 104 & $512 \times 512$ & 2.5 & 120 & 265 \\
\hline
\end{tabular}
$L C P$ with rotation-invariant uniform patters at $(2,16)$ neighbourhood size

Table 1. Data acquisition details of LIDC-IDRI-CT image database. 


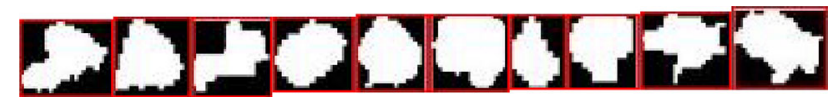

Figure 6. Sample images from LIDC-IDRI-CT image database.

\section{Experimental results and discussion}

The performance of the various methods is analysed for biomedical image retrieval by conducting experiments on medical database. The database includes CT images with region of interest (ROI). The results show that $L C P$ yields significant improvement in terms of $A R P$ and $A R R$ as compared with $L B P$ and other existing texture descriptors. In all the experiments, each image in the database is chosen as the query image. For each query image, the system collects $n$ database images $X=\left(x_{1}, x_{2}, \ldots, x_{n}\right)$, with the shortest image matching distance (Eq. (14)). If $x_{i} ; i=1,2$, $\ldots, n$ are associated with the same category of the query image, we can say that the system has correctly matched the desired.

\subsection{Experiment set 1}

Due to high robustness and reliable discriminative information of $L C P$, it has given overwhelming retrieval performance over $L B P$. To evaluate the performance of $L C P$ method, experiments are conducted on the images collected from Lung Image Database Consortium and Image Database Resource Initiative (LIDC-IDRI), which is a public lung image database of CT scans provided by Kascic [35] and NEMA-CT online image database [36]. The database is separated into 84 cases, each containing around 100-400 Digital Imaging and Communication (DICOM) images and an XML data file containing the physicians' annotations. The database contains 143 nodules having size range of $3-30 \mathrm{~mm}$ (to be manually segmented by radiologists). The CT lung images $(512 \times 512)$ from the database are converted into 'tif' image format for rapid processing with the use of Lampert's LIDC 2 image toolbox [37] (available online). Radiologists detected the locations of nodules, which have also been provided. Furthermore, ROIs were annotated manually from each slice from some patients to construct the ROI CT image database. For the experiment, 12 patient cases consisting of 75 nodules (26 benign and 49 malignant) and 229 slices have been selected. The CT scan data acquisition details are given in table 1. Figure 6 depicts the sample lung nodule images of LIDC-IDRI-CT database (one image from each patient scan).

Table 2 shows the performance of various methods on LIDC-IDRI-CT database in terms of $A R P$ by passing different query images. Figure $7 \mathrm{a}$ shows the retrieval performance in terms of $A R P$ of all the methods $\left(L B P_{1,8}^{u 2}, L B P_{1,8}^{u 2} / V A R_{1,8}, L B P_{1,8}^{r i u 2}, L B P_{1,8}^{r i u 2} / V A R_{1,8}, L C P_{1,8}^{r i u 2}\right.$, $\left.L C P_{2,8}^{\text {riu } 2}, L C P_{2,16}^{\text {riu } 2}\right)$ as a function of number of top matches. Comparison performance of the $L C P_{2,16}^{r i u 2}$ over other methods as a function of number of top matches is also given in figure $7 \mathrm{~b}$ in terms of $A R R$ on the database. In the results, $L C P_{2,16}^{\text {riu } 2}$ and $L C P_{2,8}^{\text {riu } 2}$ show better performance than that of $L B P$ on different values of $P, R$ because $L C P$ s also extract more microscopic pattern information from this database. Figure $7 \mathrm{~b}$ and table 3 summarize the performance of $L C P$ with $L B P$ on different values of $P$ and $R$. From here, it is observed that $L C P$ values on the specific radius $R=2$ and $P=16$ show overwhelming response than $L C P_{1,8}^{\text {riu } 2}, L C P_{2,8}^{\text {riu } 2}$. Figure 8 illustrates three query results of $L B P$ and $L C P$ by considering 10 top matches on LIDC-IDRI-CT database. In each result, top row presents the query image.

4.1a LCP over other existing methods on LIDC-IDRI-CT: Further, the results of $L C P$ method over other existing methods ( $L P Q, L Q P, B G P, B S I F, G L C M, W L D, S I F T)$ are compared and it is observed that it gives outstanding

Table 2. Performance of various methods by passing different query images in terms of $A R P$ on LIDC-IDRI-CT database.

\begin{tabular}{|c|c|c|c|c|c|c|c|}
\hline \multirow[b]{2}{*}{ Query images } & \multicolumn{7}{|c|}{$A R P$} \\
\hline & $L B P_{1,8}^{u 2}$ & $L B P_{1,8}^{u 2} / V A R_{1,8}$ & $L B P_{1,8}^{r i u 2}$ & $L B P_{1,8}^{r i u 2} / V A R_{1,8}$ & $L C P_{1,8}^{r i u 2}$ & $L C P_{2,8}^{r i u 2}$ & $L C P_{2,16}^{r i u 2}$ \\
\hline Query image 1 & 0.7032 & 0.7036 & 0.7823 & 0.7823 & 0.7462 & 1.0000 & 1.0000 \\
\hline Query image 2 & 0.8274 & 1.0000 & 0.9765 & 0.9765 & 1.0000 & 1.0000 & 1.0000 \\
\hline Query image 3 & 0.7143 & 0.4585 & 0.5022 & 0.5022 & 1.0000 & 0.7917 & 0.5423 \\
\hline Query image 4 & 0.6111 & 0.3674 & 0.5889 & 0.5889 & 0.7226 & 0.7481 & 1.0000 \\
\hline Query image 5 & 1.0000 & 0.6428 & 1.0000 & 1.0000 & 0.2111 & 0.7024 & 1.0000 \\
\hline Query image 6 & 0.9468 & 0.6188 & 0.7972 & 0.7972 & 0.7885 & 0.7024 & 1.0000 \\
\hline Query image 7 & 0.8553 & 0.5954 & 0.7167 & 0.7167 & 0.8357 & 0.9107 & 1.0000 \\
\hline Query image 8 & 1.0000 & 0.7889 & 0.9283 & 0.9283 & 0.1944 & 0.7254 & 1.0000 \\
\hline Query image 9 & 0.3696 & 0.2917 & 0.2897 & 0.2897 & 0.8486 & 0.2500 & 0.8523 \\
\hline Query image 10 & 0.1000 & 0.2500 & 0 & 0 & 0.8972 & 1.0000 & 1.0000 \\
\hline
\end{tabular}




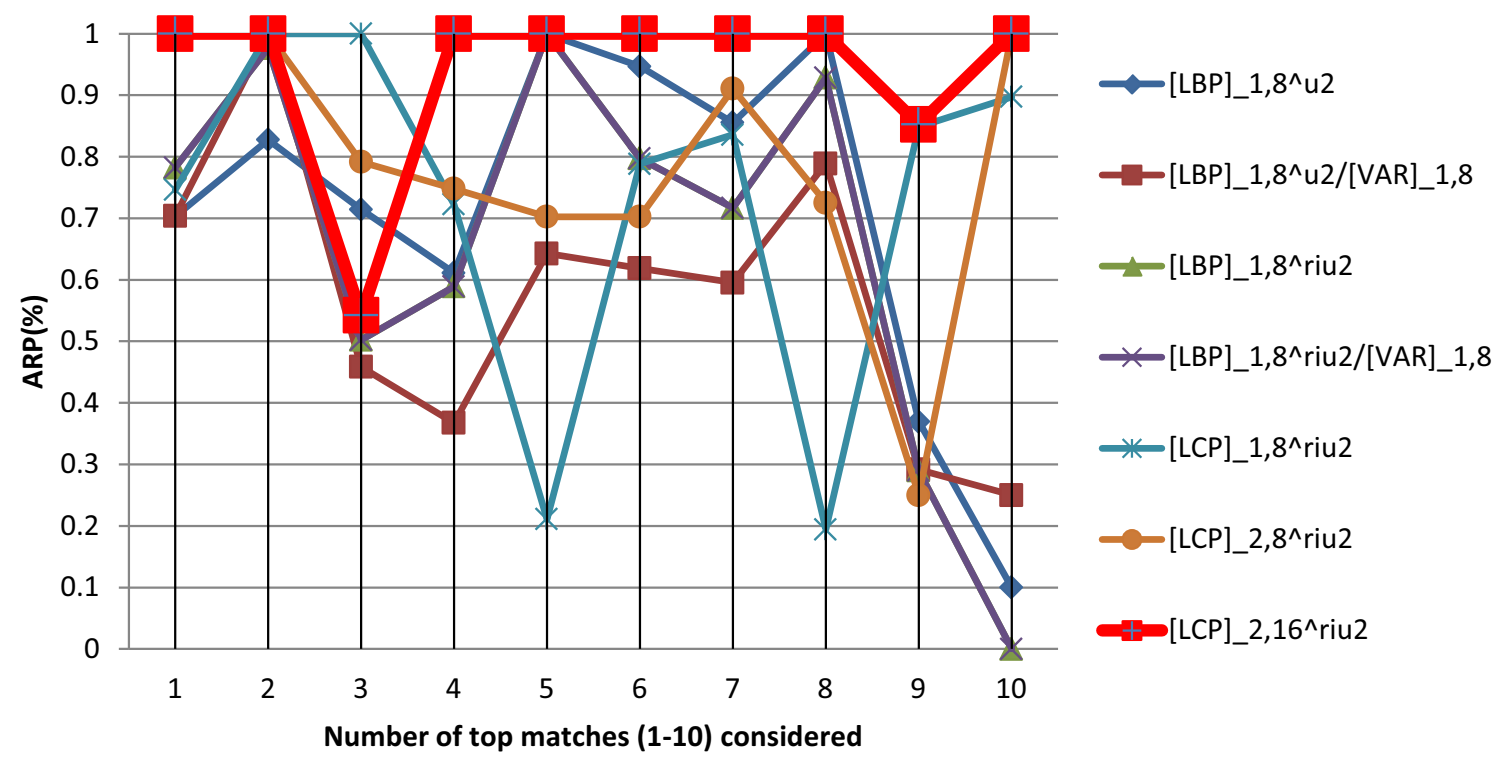

(a)

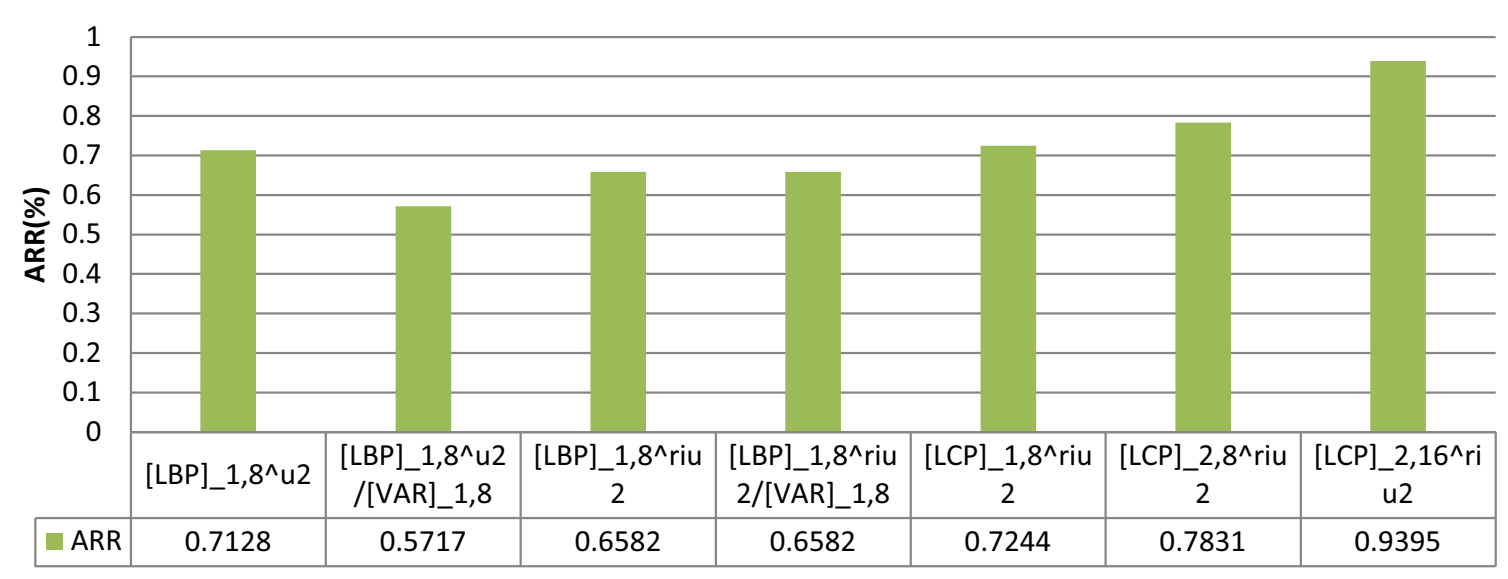

(b)

Figure 7. (a) Graphical performance analysis of various methods as function of number of top matches (1-10) in terms of ARP and (b) comparison of the $L C P_{2,16}^{\text {riu } 2}$ over other methods as function of number of top matches (1-10) in terms of ARR on LIDC-IDRI-CT database.

Table 3. Performance analysis of various methods in terms of $A R R$ on LIDC-IDRI-CT database.

\begin{tabular}{lc}
\hline Method & $A R R$ \\
\hline$L B P_{1,8}^{u 2}$ & 0.7128 \\
$L B P_{1,8}^{u 2} / V A R_{1,8}$ & 0.5717 \\
$L B P_{1,8}^{\text {riu2 }}$ & 0.6582 \\
$L B P_{1,8}^{\text {riu2 }} / V A R_{1,8}$ & 0.6582 \\
$L C P_{1,8}^{\text {riu2 }}$ & 0.7244 \\
$L C P_{2,8}^{\text {riu2 }}$ & 0.7831 \\
$L C P_{2,16}^{\text {riu2 }}$ & 0.9395 \\
\hline
\end{tabular}

performance. The performance of $L C P$ over other existing texture descriptors is shown in tables 4 and 5 and figure 9.

\subsection{Feature vector length $V / S$ performance}

Table 6 shows the feature vector length for a given query image using $\quad L B P_{1,8}^{u 2}, L B P_{1,8}^{u 2} / V A R_{1,8}, L B P_{1,8}^{\text {riu2 }}, L B P_{1,8}^{\text {riu } 2} / V A R_{1,8}$, $L C P_{1,8}^{r i u 2}, L C P_{2,8}^{r i u 2}, L C P_{2,16}^{r i u 2}$. The experimentation is carried out on a core 2 Quad computer with $2.66 \mathrm{GHz}, 4 \mathrm{~GB}$ of memory and all methods are implemented on the MATLAB software. From table 6 , it is clear that the feature vector length of 


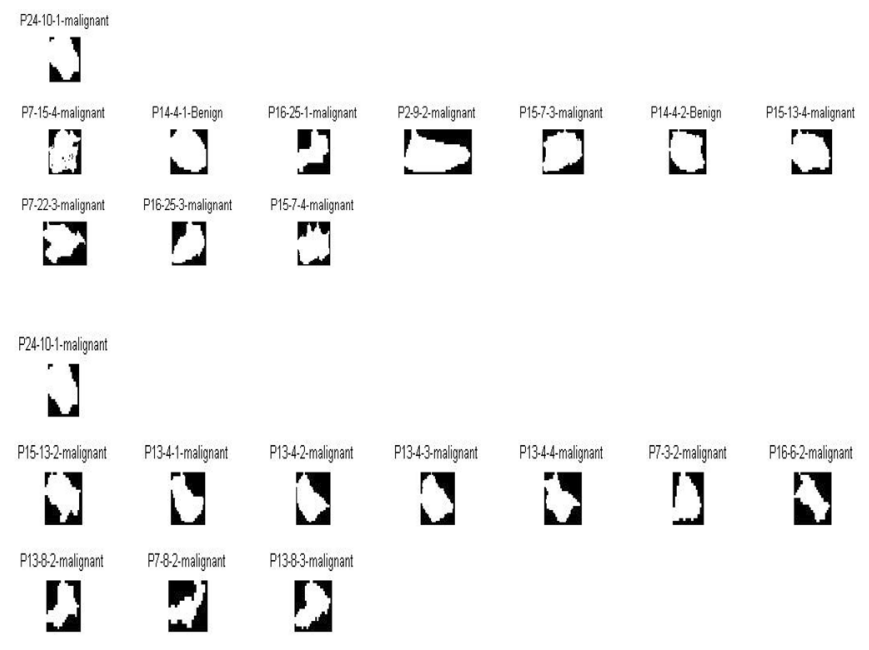

(a) query image 1

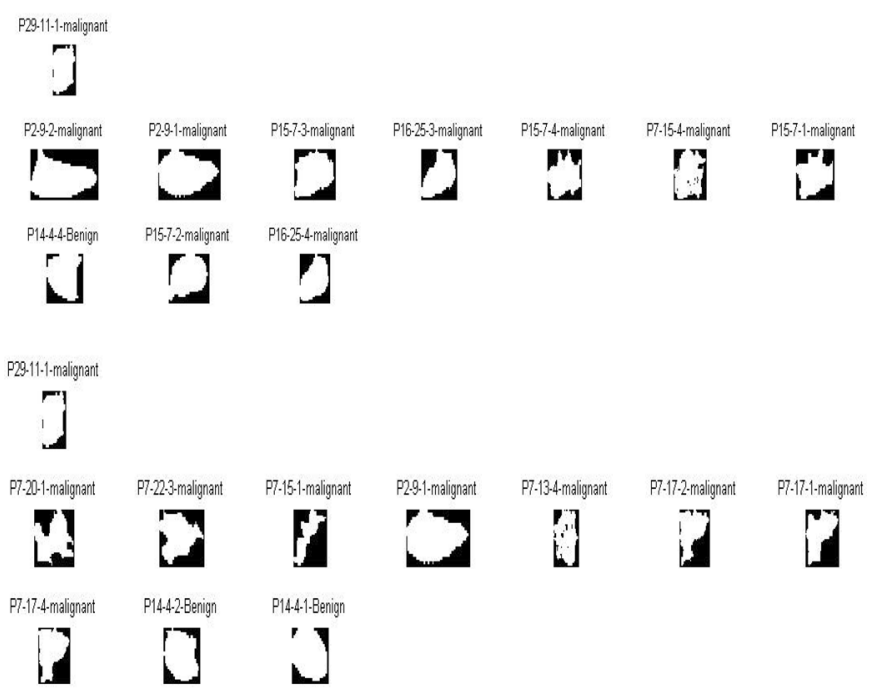

(b) query image 2

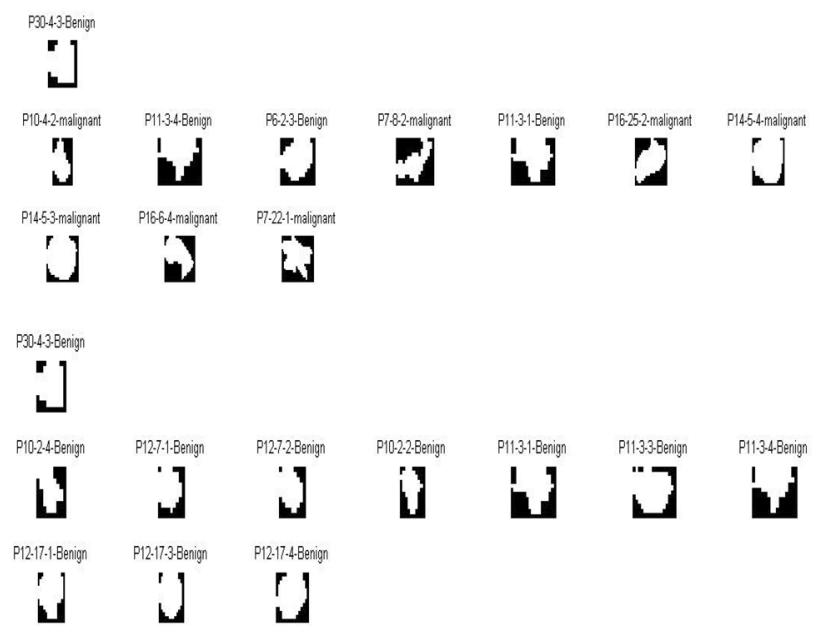

(c) query image 3

Figure 8. Query results on LIDC-IDRI-CT database (a-c) through $L B P$ and $L C P$, respectively. 


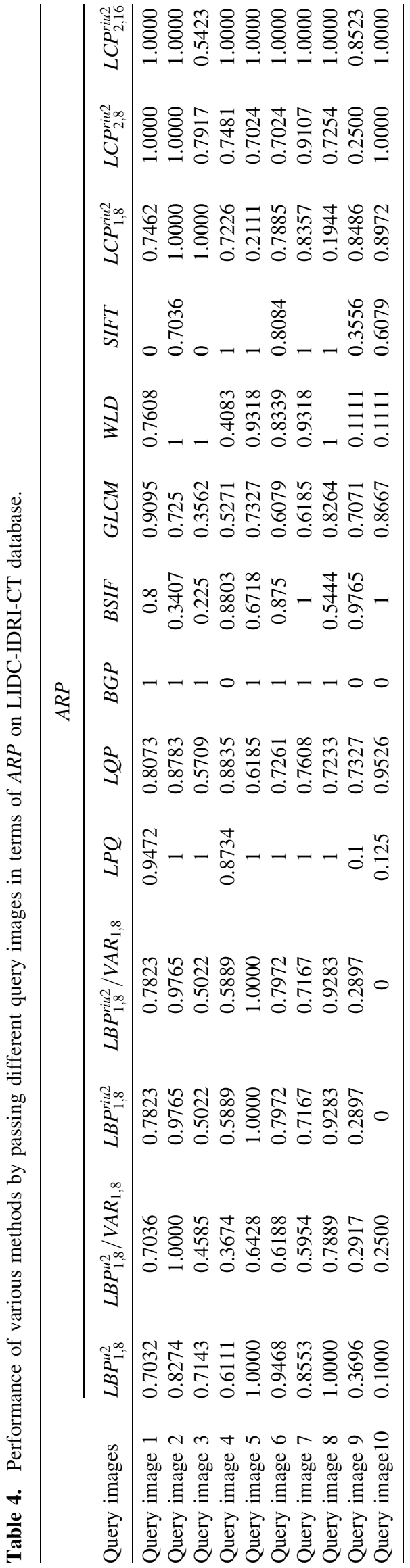

Table 5. Performance analysis of various methods in terms of $A R R$ on LIDC-IDRI-CT database.

\begin{tabular}{lc}
\hline Method & $A R R$ \\
\hline$L B P_{1,8}^{u 2}$ & 0.7128 \\
$L B P_{1,8}^{u 2} / V A R_{1,8}$ & 0.5717 \\
$L B P_{1,8}^{\text {riu }}$ & 0.6582 \\
$L B P_{1,8}^{r i u 2} / V A R_{1,8}$ & 0.6582 \\
$L P Q$ & 0.8046 \\
$L Q P$ & 0.7654 \\
$B G P$ & 0.7000 \\
$B S I F$ & 0.7314 \\
$G L C M$ & 0.6877 \\
$W L D$ & 0.7089 \\
$S I F T$ & 0.6475 \\
$L C P_{1,8}^{r i u 2}$ & 0.7244 \\
$L C P_{2,8}^{\text {riu }}$ & 0.7831 \\
$L C P_{2,16}^{\text {riu }}$ & 0.9395 \\
\hline
\end{tabular}

Table 6. Feature vector length of query image using various methods.

\begin{tabular}{lc}
\hline Method & Feature vector length \\
\hline$L B P_{1,8}^{u 2}$ & 59 \\
$L B P_{1,8}^{u 2} / V A R_{1,8}$ & 59 \\
$L B P_{1,8}^{r i u 2}$ & 38 \\
$L B P_{1,8}^{\text {riu }} / V A R_{1,8}$ & 38 \\
$L C P_{1,8}^{\text {riu2 }}$ & 81 \\
$L C P_{2,8}^{\text {riu }}$ & 81 \\
$L C P_{2,16}^{\text {riu2 }}$ & 289 \\
\hline
\end{tabular}

$L C P_{2,16}^{r i w 2}$ is more as compared with $L B P$, as it outperforms in terms of $A R P$ and $A R R$ on biomedical database.

\section{Conclusions}

In this paper, the applicability of existing $L C P$ image indexing and retrieval algorithm is analysed. The $L C P$ explores multi-channel discriminative information of both the $\mathrm{MiC}$ and local structures of an image. The performance of the LCP method is also analysed with different configuration pattern values on different radii and neighbourhood sizes. The performance improvement has been compared with the $L B P$ on benchmark biomedical image database. The result shows a significant improvement in terms of precision, recall, $A R R$ and $A R P$ as compared with $L B P$ and other existing texture descriptors on LIDC-IDRI-CT database.

The generality of the $L C P$ descriptor is validated across one benchmark biomedical database. In future experiments, the same descriptor can be compared in other biomedical or ordinary datasets against state of the art descriptors. 

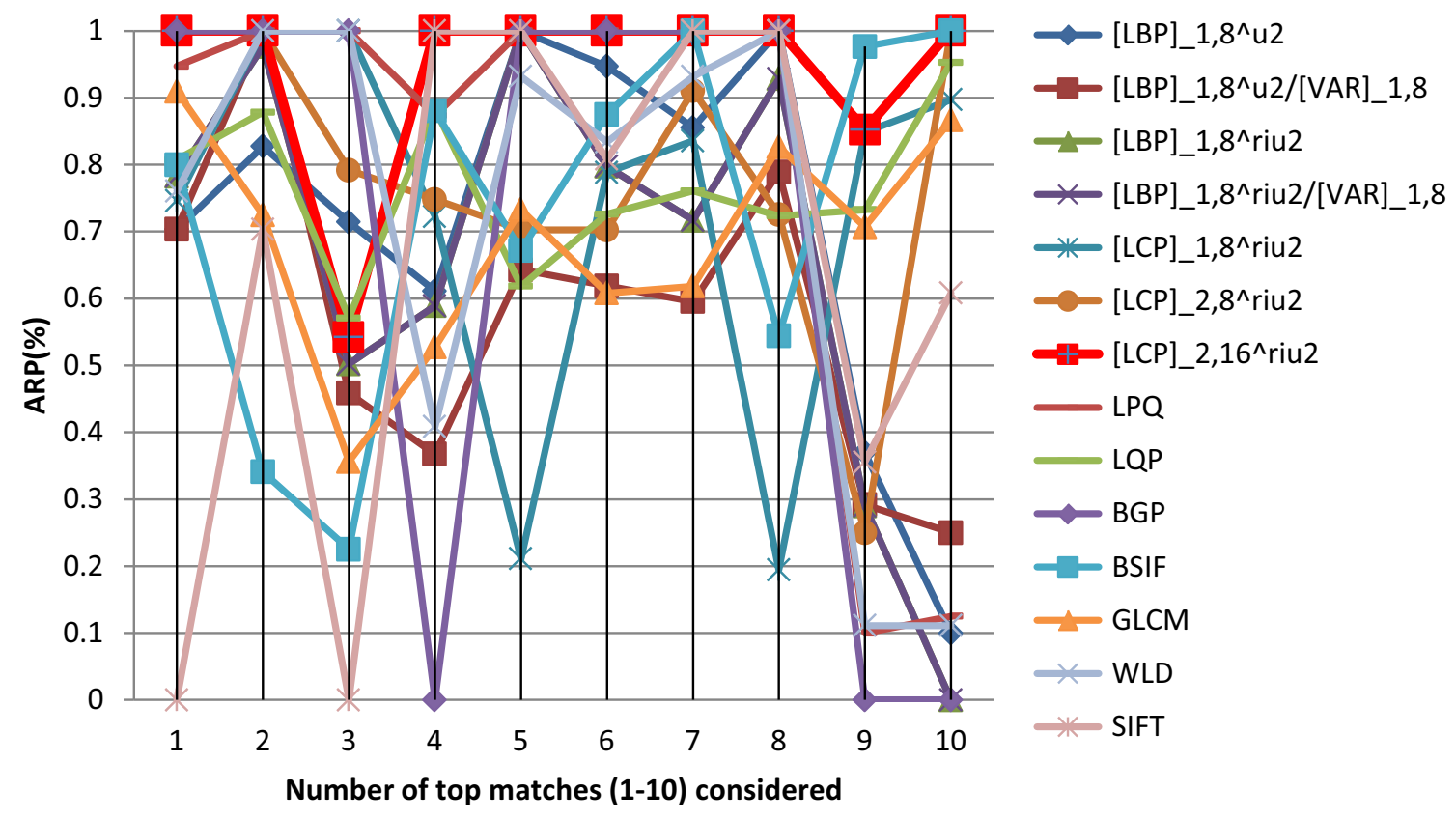

(a)

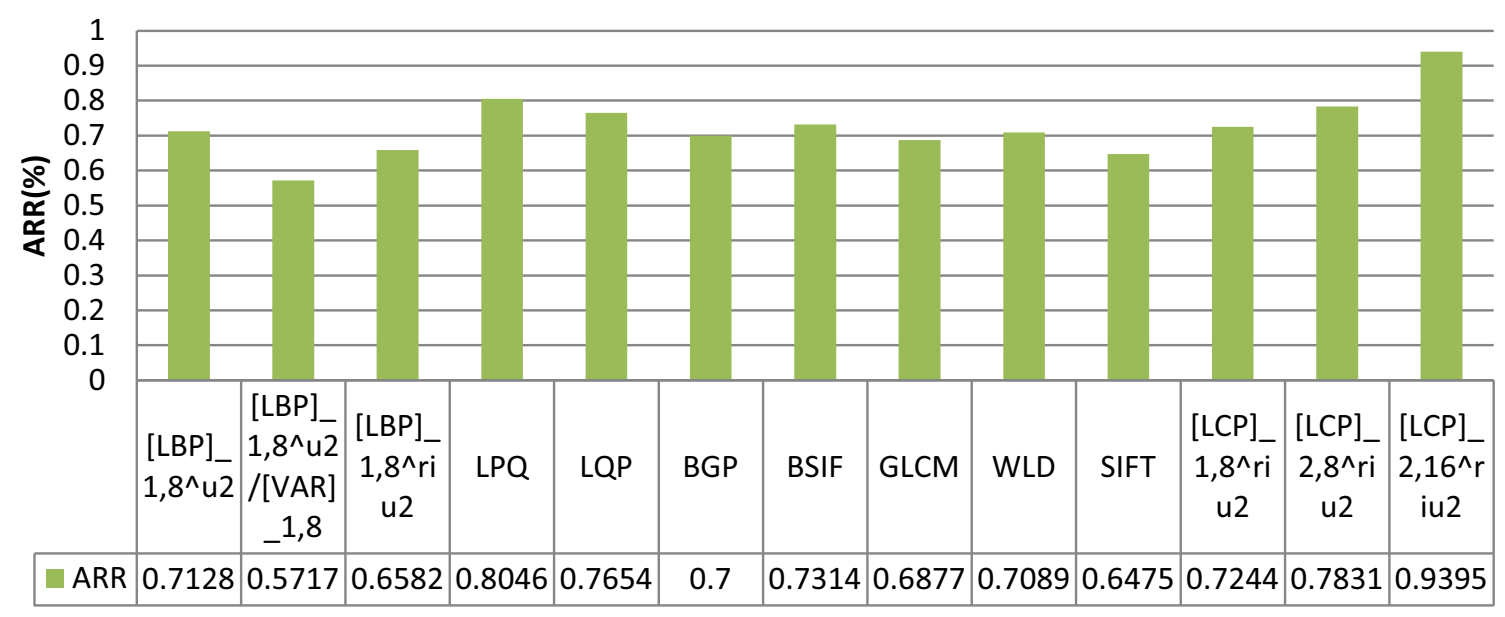

(b)

Figure 9. (a) Graphical performance analysis of various methods as function of number of top matches (1-10) in terms of ARP and (b) comparison of the $L C P_{2,16}^{\text {riu } 2}$ over other existing texture descriptors as function of number of top matches (1-10) in terms of ARR on LIDCIDRI-CT database.

\section{Acknowledgements}

We wish to express our appreciation to the Lung Image Database Consortium, who contributed to the creation of the LIDC collection as an independent testing dataset. We would like to thank anonymous reviewers for insightful comments and valuable suggestions to improve the quality of this manuscript.

\section{References}

[1] Giuliano P, Pietro Z and Guido M C 2013 Combining color and shape descriptors for 3D model retrieval. Sig. Process. Imag. Commun. 28: 608-623

[2] Michalis L, Apostolos A, Dimitrios R and Petros D 2013 Multimedia search and retrieval using multimodal annotation 
propagation and indexing techniques. Sig. Process. Imag. Commun. 28: 351-367

[3] Vo A P N and Soontorn O 2010 A study of relative phase in complex wavelet domain: property, statistics and applications in texture image retrieval and segmentation. Sig. Process. Imag. Commun. 25: 28-46

[4] Gianluca F, Skjalg L and Massimo B 2013 Selection of local features for visual search. Sig. Process. Imag. Commun. 28: 311-322

[5] Sihyoung L, Wesley D N and Yong M R 2010 Tag refinement in an image folksonomy using visual similarity and tag co-occurrence statistics. Sig. Process. Imag. Commun. 25: 761-773

[6] Yue G, Qionghai D, Meng W and Naiyao Z 2011 3D model retrieval using weighted bipartite graph matching. Sig. Process. Imag. Commun. 26: 39-47

[7] Manjunath K N, Renuka A and Niranjan U C 2007 Linear models of cumulative distribution function for content-based medical image retrieval. J. Med. Syst. 31: 433-443

[8] Ojala T, Pietikainen M and Harwood D 1996 A comparative study of texture measures with classification based on feature distributions. Pattern Recogn. 29(1): 51-59

[9] Ojala T, Pietikainen M and Maeenpaa T 2002 Multiresolution gray-scale and rotation invariant texture classification with local binary patterns. IEEE Trans. Pattern Anal. Mach. Intell. 24(7): 971-987

[10] Oliver A, Lladó X, Freixenet J and Martí J 2007 False positive reduction in mammographic mass detection using local binary patterns. In: Proceedings of the Medical Image Computing and Computer-Assisted Intervention (MICCAI 2007), Brisbane, Australia, Lecture Notes in Computer Science 4791. Springer, Berlin, Heidelberg, pp. 286-293

[11] Unay D and Ekin A 2008 Intensity versus texture for medical image search and retrieval. In: Proceedings of the Fifth IEEE International Symposium on Biomedical Imaging: From Nano to Macro (ISBI 2008), pp. 241-244

[12] Keramidas E G, Iakovidis D K, Maroulis D and Dimitropoulos N 2008 Thyroid texture representation via noise resistant image features. In: Proceedings of the 21st IEEE International Symposium on Computer-Based Medical Systems (CBMS 2008), pp. 560-565

[13] Nanni L and Lumini A 2008 A reliable method for cell phenotype image classification. Artif. Intell. Med. 43(2): 87-97

[14] Nanni L and Lumini A 2008 Ensemble of neural networks for automated cell phenotype image classification. In: Biomedical image analysis and machine learning technologies: applications and techniques [chapter in vision 2008], pp. 234-259

[15] Nanni L and Lumini A 2007 Region Boost learning for 2D + 3D based face recognition. Pattern Recogn. Lett. 28(15): 2063-2070

[16] Ahonen T, Hadid A and Pietikainen M 2006 Face description with local binary patterns: application to face recognition. IEEE Trans. Pattern Anal. Mach. Intell. 28(12): 2037-2041

[17] Guo Z, Zhang L and Zhang D 2010 Rotation invariant texture classification using LBP variance with global matching. Pattern Recogn. 43: 706-716

[18] Nanni L and Lumini A 2008 Local binary patterns for a hybrid fingerprint matcher. Pattern Recogn. 11: 3461-3466

[19] Shang X and Veldhuis R 2007 Local absolute binary patterns as image preprocessing for grip-pattern recognition in smart gun. In: Proceedings of the First IEEE International Conference on Biometrics: Theory, Applications, and Systems, pp. 1-6

[20] Guo Y, Zhao G and Pietikäinen M 2011 Texture classification using a linear configuration model based descriptor. In: Proceedings of the British Machine Vision Conference (BMVC 2011), Dundee, UK, pp. 119.1-119.10

[21] Murala S, Maheshwari R P and Balasubramanian R 2012 Directional binary wavelet patterns for biomedical image indexing and retrieval. J. Med. Syst. 36(5): 2865-2879

[22] Murala S, Maheshwari R P and Balasubramanian R 2012 Directional local extrema patterns: a new descriptor for content based image retrieval. Int. J. Multimedia Inf. Retr. 1(3): 191-203

[23] Murala S, Maheshwari R P and Balasubramanian R 2012 Local maximum edge binary patterns: a new descriptor for image retrieval and object tracking. Sig. Process. 92: 1467-1479

[24] Murala S, Maheshwari R P and Balasubramanian R 2012 Local tetra patterns: new feature descriptor for content based image retrieval. IEEE Trans. Imag. Process. 21(5): 2874-2886

[25] Murala S and Jonathan W Q 2013 Local ternary co-occurrence patterns: a new feature descriptor for MRI and CT image retrieval. Neurocomputing 119(7): 399-412

[26] Murala S and Jonathan W Q 2013 Peak valley edge patterns: a new descriptor for biomedical image indexing and retrieval. In: Proceedings of the IEEE Conference on Computer Vision and Pattern Recognition Workshops (CVPRW), pp. 444-449

[27] Roweis S and Saul L 2000 Nonlinear dimensionality reduction by locally linear embedding. Science 290: 2323-2326

[28] Ojansivu V and Heikkila J 2008 Blur insensitive texture classification using local phase quantization. In: Proceedings of Image and Signal Processing (ICISP), Lecture Notes in Computer Science 5099, pp. 236-243

[29] Nanni L, Lumini A and Brahnam S 2010 Local binary patterns variants as texture descriptors for medical image analysis. Artif. Intell. Med. 49(2): 117-125

[30] Zhang L, Zhou Z and Li H 2012 Binary Gabor pattern: an efficient and robust descriptor for texture classification. In: Proceedings of the 19th IEEE International Conference on Image Processing (ICIP), pp. 81-84

[31] Kannala J and Rahtu E 2012 BSIF: binarized statistical image features. In: Proceedings of the 21st International Conference on Pattern Recognition (ICPR), pp. 1363-1366

[32] Chen J, Shan S, He C, Zhao G, Pietikäinen M, Chen X and Gao W 2009 WLD: a robust local image descriptor. IEEE Trans. Pattern Anal. Mach. Intell. 32(9): 1705-1720

[33] Lowe D 2004 Distinctive image features from scale-invariant keypoints. Int. J. Comput. Vis. 60(2): 91-110

[34] Haralick R M, Shanmugam K and Dinstein I 1973 Textural features for image classification. IEEE Trans. Syst. Man Cybern. 3: 610-621

[35] Kascic E 2011 NBIA - National Cancer Imaging Archive NCIA (version 4.0): the NCI's repository for DICOM-based images (https://cabig.nci.nih.gov/tools/NCIA)

[36] NEMA-CT image database 2012 [online] Available: ftp:// medical.nema.org/medical/Dicom/Multiframe/

[37] Lampert T LIDC 2 Image Toolbox (Matlab) [online]. Available: https://wiki.cancerimagingarchive.net/display/ Public/LungImageDatabaseConsortium 